\title{
Chiral Magneto-Electrochemistry
}

\author{
Anup Kumar ${ }^{1}$, Prakash Chandra Mondal 2 (i) and Claudio Fontanesi ${ }^{3, *}$ \\ 1 Department of Chemical and Biological Physics, Weizmann Institute of Science, Rehovot 76100, Israel; \\ anup12271982@gmail.com \\ 2 Institute of Molecular Science (ICMOL), University of Valencia, 46980 Paterna, Spain; mondalpc@gmail.com \\ 3 Department of Engineering “Enzo Ferrari”, University of Modena and Reggio Emilia, Via Vivarelli 10, \\ 41125 Modena, Italy \\ * Correspondence: claudio.fontanesi@unimore.it
}

Received: 10 June 2018; Accepted: 13 August 2018; Published: 18 August 2018

\begin{abstract}
Magneto-electrochemistry (MEC) is a unique paradigm in science, where electrochemical experiments are carried out as a function of an applied magnetic field, creating a new horizon of potential scientific interest and technological applications. Over time, detailed understanding of this research domain was developed to identify and rationalize the possible effects exerted by a magnetic field on the various microscopic processes occurring in an electrochemical system. Notably, until a few years ago, the role of spin was not taken into account in the field of magneto-electrochemistry. Remarkably, recent experimental studies reveal that electron transmission through chiral molecules is spin selective and this effect has been referred to as the chiral-induced spin selectivity (CISS) effect. Spin-dependent electrochemistry originates from the implementation of the CISS effect in electrochemistry, where the magnetic field is used to obtain spin-polarized currents (using ferromagnetic electrodes) or, conversely, a magnetic field is obtained as the result of spin accumulation.
\end{abstract}

Keywords: magneto-electrochemistry; CISS; spin; chirality; spin-dependent electrochemistry

\section{Introduction}

Magneto-electrochemistry (MEC) combines two distinct paradigms, i.e., electrochemistry and magnetism, where synergic effects are possible upon changing directions of the superimposition of two independent quantities, i.e., the electric and magnetic fields, causing complex hydrodynamics conditions in the cell, as well as the emergence of unique electronic properties in both bulk reagents and electrodes [1]. Indeed, the effect of magnetic field intensity and direction on electrochemical processes, in a variety of ways and experimental configurations, was first observed by Michael Faraday [2].

Quite elegant and interesting papers are present in the literature aiming to sort out the effect of a magnetic field on both bulk and interfacial properties in electrochemical systems. From this view, quite interesting results report on the "chiral texture" observed in electrodeposited films grown under the influence of an applied magnetic field, including metals ( $\mathrm{Cu}, \mathrm{Ag}$, Fe etc.), alloys, and conducting polymers (polyaniline, etc.). Such chiral structures are useful in technological applications like enantioselective recognition processes [3,4].

So far most of the studies in the literature have used an external magnetic field to generate chiral structures or special hydrodynamic conditions. Of late, Naaman et al., discovered a revolutionary "chiral-induced spin selectivity (CISS)" effect: the electron transmission through chiral systems depends on the spin, giving rise to a net magnetic moment (with the associated magnetic field) ([5] and references therein cited). The idea was recently demonstrated via an innovative Hall device that serves as the working electrode in an electrochemical cell, and is able to give information on the correlation of spin selectivity and the electrochemical process without an externally applied magnetic field [6]. 
This article aims to give an overview of experimental findings obtained by combining magneto-electrochemical systems and chiral architectures, like in 2D metallic or organic electrodeposition under an external imposed magnetic field, and their enantioselective recognition/adsorption processes [7]. We also focus on highlighting the exciting results obtained by spin-specific electron transmission through chiral interfaces in electrochemical systems, namely, spin-dependent electrochemistry [8].

The perspective is that these two disjoined fields of magnetochemistry can find a possible way of interaction, for instance, the use of chiral electrodeposited metals or alloys in spin-dependent electrochemistry, thus allowing for the observation of unexplored synergic effects.

\section{Magnetic Effect on the Electrolyte Solution}

The great majority of magneto-electrochemistry experiments can be rationalized by using relationships well established in classical electromagnetism. For instance, the electrolytic solution conductivity is due to the migration of ions under an applied electric field E. The relevant electrostatic force acting on the ions of charge $q$ is given by Equation (1):

$$
F=q E,
$$

and the effect of a magnetic field $B$, which yields a Lorentz type force, on the moving ion of charge $q$, is accounted for by Equation (2) [8,9]:

$$
F=q(E+v \times B),
$$

where $v$ is the speed of the migrating ion in solution. Equation (2) gives due reason to the deviation of the motion of ions from a simple linear trajectory: compare the well-known Hall effect in a semiconductor [9]. This effect is referred to as the magneto-hydrodynamics (MHD) force [10]. Indeed, in the MHD theory the effect of a uniform magnetic field on a conductive fluid, like the situation found in an electrochemical system, differs from the effect of a magnetic field gradient in simple electrostatics. Equation (3) represents the force acting on the moving ion in a nonuniform magnetic field, which is a more complex situation with respect to vacuum [1]:

$$
\vec{F}_{m a g}=\frac{\chi_{m} B^{2} \vec{\nabla}_{c}}{2 \mu_{0}}+\frac{\chi_{m} c_{0} B \vec{\nabla} B}{\mu_{0}} .
$$

In Equation (3), $\chi_{m}$ is the molar susceptibility, $\mu_{0}$ is the free space permeability, and $c_{0}$ is the bulk concentration. This expression of $\vec{F}_{m a g}$ consists of two terms: the first term of the right-hand side of Equation (3) is the paramagnetic gradient force (which depends on the gradient of the concentration); the second term in Equation (3) is the field gradient force (which can be significant even in the case of a non-uniform magnetic field, such as can be found close to the rough surface of a ferromagnetic electrode). Note that Equations (1) to (3) are part of a classical approach when spin effects or symmetry-related effects, like spin-chirality interaction, are accounted for. Indeed, magnetic field effects on the bulk electrolyte mass transport process can be rationalized within such a classical-based approach [11].

\section{Electrodeposition of Chiral Structures Induced by a Magnetic Field}

Application of a magnetic field during the process of electrodeposition of metals and alloys, as well as in electropolymerization reactions, gives rise to complex texture and morphology, a subject of great interest for material scientists. In general, materials electrodeposited under application of a magnetic field are reported to show chiral texture, either with clockwise or anticlockwise handedness, depending on the magnetic field orientation with respect to the electrode surface [12]. Mhíocháin et al. showed that zinc fractal-like shapes are grown around a point cathode during zinc electrodeposition in a flat, horizontal, electrochemical cell [13]. When the zinc is electrodeposited under an applied 
perpendicular magnetic field a chiral spiral structure is developed; opposite handedness of the zinc deposit is obtained on switching the magnetic field direction (Figure 1).

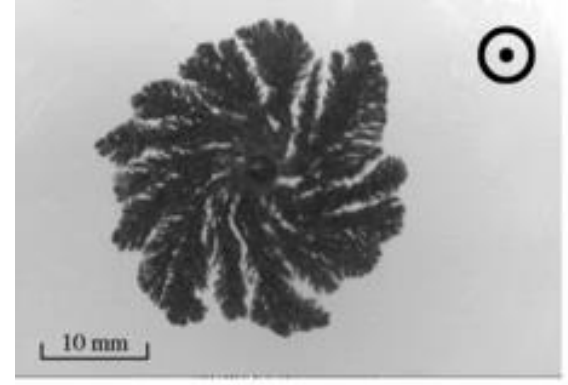

(a)

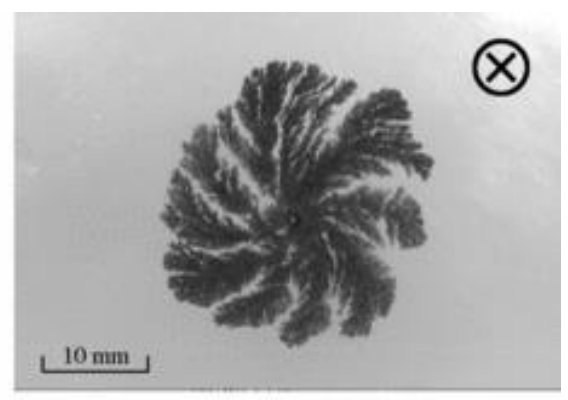

(b)

Figure 1. Zinc electrodeposited from a $0.1 \mathrm{M} \mathrm{ZnSO}_{4}$ aqueous solution at $10 \mathrm{~V}$, in a flat horizontal cell, with $0.35 \mathrm{~T}$ magnetic field intensity. The latter is applied (a) vertically upward or (b) vertically downward (Reproduced with permission from [13]. The American Physical Society, 2004).

In the case of the electrodeposition of nickel-iron and cobalt-iron alloys, X-ray diffraction (XRD) and scanning electron microscopy (SEM) analyses proved the occurrence of modifications in the alloy chemical composition, as well as in the surface roughness, induced by the presence of the magnetic field [14]. Also, the electropolymerization of organic compounds shows a magnetic field dependence: chiral manifestations have been observed during polyaniline electropolymerization in high magnetic fields [15]. It is important to emphasize that chiral surfaces can act as enantioselective catalysts able to discriminate two enantiomeric forms [16] and to be obtained in a variety of ways such as by adsorbing chiral molecules [17] or chiral high-index electrode surfaces [18].

Switzer et al. proposed a method to produce a chiral surface of copper oxide through electrodeposition in the presence of chiral tartrate anion; the chirality of the copper oxide surface can be tuned by changing the chirality of the tartrate ion in solution [19]. In order to analyze the surface chirality, the electrochemical oxidation of $(\mathrm{S}, \mathrm{S})$ - and $(\mathrm{R}, \mathrm{R})$-tartrate was carried out. Figure $2 \mathrm{a}$ shows the oxidation of $(\mathrm{S}, \mathrm{S})$ - and $(\mathrm{R}, \mathrm{R})$-tartrate on $\mathrm{CuO}$ electrodes that were deposited from $\mathrm{Cu}(\mathrm{II})$ $(\mathrm{S}, \mathrm{S})$-tartrate and $\mathrm{Cu}(\mathrm{II})(\mathrm{R}, \mathrm{R})$-tartrate (Figure 2b). Notably, (S)-CuO film grown in (S,S)-tartrate is more active for the oxidation of the (S,S)-tartrate. On the contrary, $(\mathrm{R})-\mathrm{CuO}$ film is found active for $(R, R)$-tartrate. A control experiment carried out on the achiral surface shows no selectivity for the oxidation of enantiomers (Figure 2c). $\mathrm{CuO}$ has been also reported to be an efficient electrocatalyst for the oxidation of carbohydrates, amino acids, simple alcohols, aliphatic diols, and alkyl polyethoxy alcohol detergents [20]. Similarly, Hinds et al. studied the effect of a static magnetic field on the electrolysis of copper in aqueous solution; a significant enhancement of the electrodeposition rate (up to $300 \%$ ) was observed in highly concentrated $\mathrm{CuSO}_{4}$ solution at low $\mathrm{pH}$ [21]. 


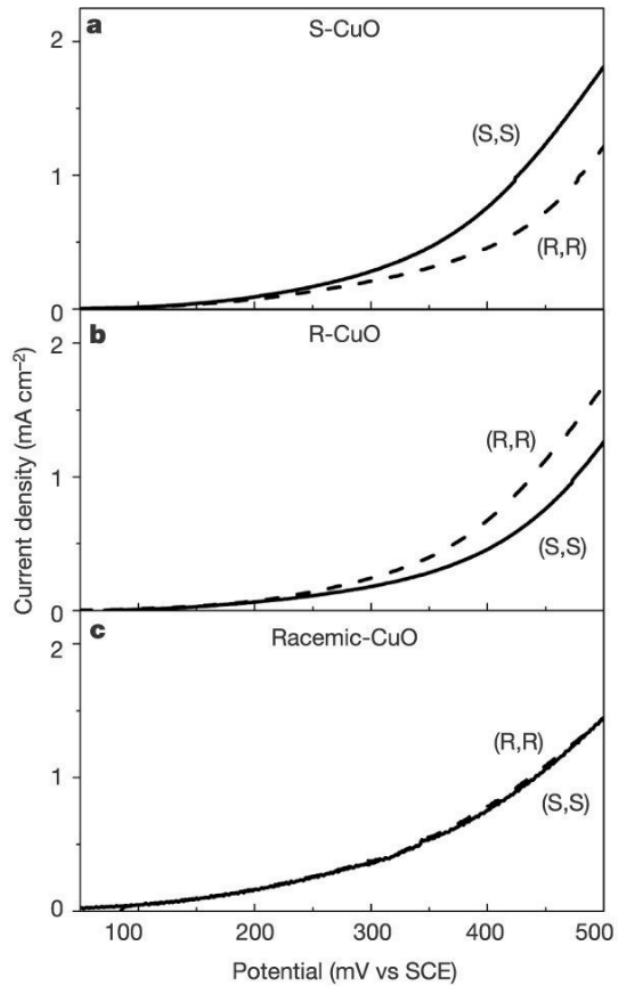

Figure 2. Enantio-recognition by electrodeposited chiral $\mathrm{CuO}$. Electrocatalytic activity of chiral electrodes (a) (S)-CuO, (b) (R)-CuO, and (c) (racemic)-CuO films towards oxidation of (R,R) and $(\mathrm{S}, \mathrm{S})$ tartaric acid (Reproduced with permission from [19], Nature Publishing Group, 2003).

Indeed, in a flat horizontal cell, dense radial growth is obtained in the absence of an applied magnetic field (Figure 3). When a magnetic field is applied perpendicular to the plane of the cell, a branched chiral spiral pattern forms (compare Figure 3b). The spiral pattern reverses handedness upon flipping of the field direction (Figure 3c), while a parallel magnetic field applied parallel to the plane of the cell produces a stringy asymmetrical deposit (Figure 3d), predominantly in the direction where the Lorentz force and the gravitational force act downwards [21].
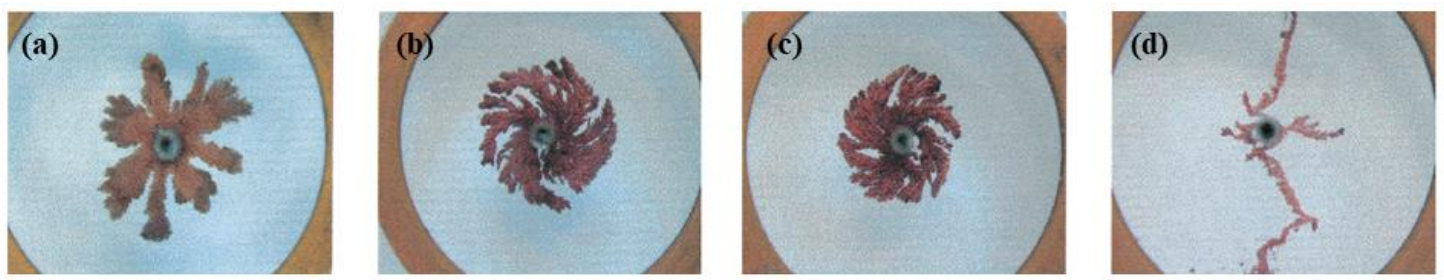

Figure 3. Morphology of copper electrodeposits grown around a central cathode in a horizontal flat circular cell (a) in zero applied field, (b) $0.4 \mathrm{~T}$ vertically upward, (c) $0.4 \mathrm{~T}$ vertically downward, and (d) $1 \mathrm{~T}$ horizontally (Reproduced with permission from [21]. American Chemical Society, 2001).

Similarly, chiral conducting polymer in the specific case of polyaniline doped with $(+)$ or (-)-camphor-10-sulfonate (CSA-) is able to recognize, used as an amperometric sensor, L- and D-ascorbic acid (AA) [15].

Yutthalekha et al. exploited mesoporous chiral metal structures for inducing asymmetry in the electrosynthesis of mandelic acid [22]. The chiral-encoded mesoporous metal, produced via electrochemical reduction of platinum salts in the presence of both a liquid crystal phase and the chiral template molecule, retains the chirality of the medium after the removal of the template. Significant 
enantiomeric excess of the (R)-enantiomer when using (R)-imprinted electrodes and vice versa for the (S)-imprinted ones was obtained [22].

\section{Hall Effect in Bulk Electrolyte}

The observation of Hall voltage in electrolytic solution is considered to be a quite controversial topic, and is an effect first shown by Roiti way back in 1882 yet with dedicated efforts by Frank and Hoffman [23]. A positive Hall coefficient for $\mathrm{CuSO}_{4}$ liquid electrolyte has been measured which depends on polarity and strength of current and magnetic field (compare Figure 4). The Hall coefficient increases with decreasing concentration of solute; as expected, the $\mathrm{H}^{+}$ion acts as the main charge carrier in a liquid electrolyte with carrier mobility of the order of $1 \mathrm{~cm}^{2} \mathrm{~V}^{-1} \mathrm{~s}^{-1}$.

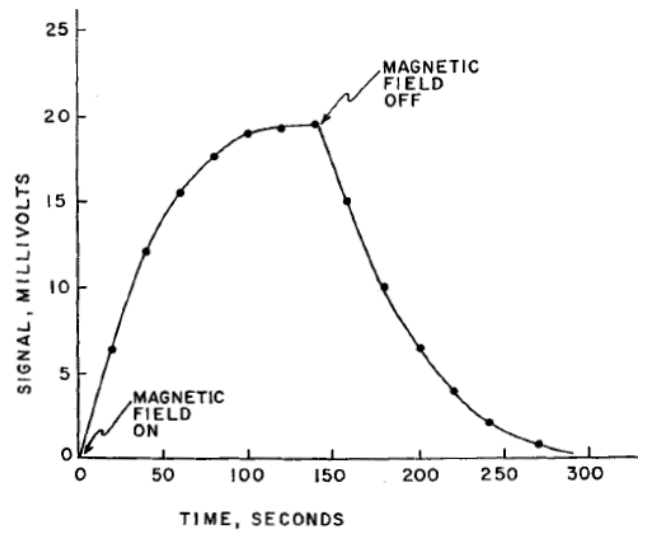

(a)

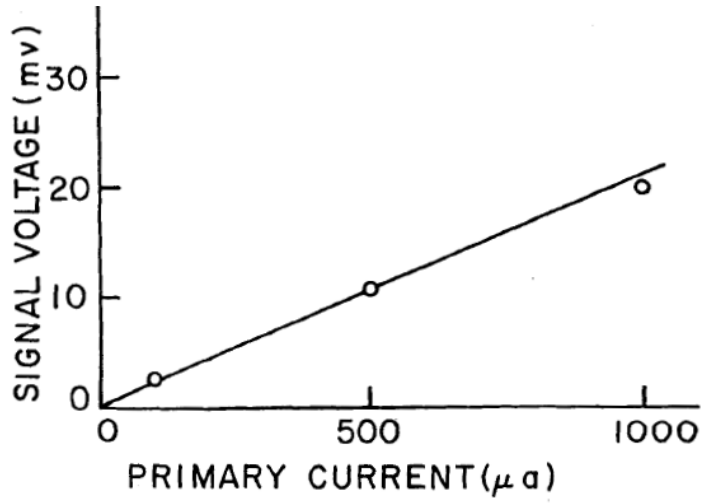

(b)

Figure 4. (a) Hall voltage measured upon application of a magnetic field as a function of time, $0.01 \mathrm{M}$ $\mathrm{CuSO}_{4}$ aqueous solution. (b) Hall voltage as a function of electrochemical current, in $0.01 \mathrm{M} \mathrm{CuSO}_{4}$ (Reproduced with permission from [23], Taylor \& Francis, 1972).

Furthermore, Hubbard and Wolynes theoretically calculated the electrohydrodynamic forces acting on an ion moving in a polarizable liquid upon application of a magnetic field [24], determining a hydrodynamic force which is about the $30 \%$ of the expected Lorentz force $\mathrm{q} / \mathrm{c}(\mathrm{v} \times B)$. This effect is found to be independent of ion size, charge, and solvent viscosity. Moreover, Newman et al. exploited the solid electrolyte $\mathrm{C}_{5} \mathrm{H}_{6} \mathrm{NAg}_{5} \mathrm{I}_{6}$ for demonstrating the ionic Hall effect; Hall potentials are found in the 10-100 $\mathrm{nV}$ range at $25{ }^{\circ} \mathrm{C}$ for $0.1-1.0 \mathrm{~T}$ [25]. The exceptionally high ionic conductivity in $\mathrm{C}_{5} \mathrm{H}_{6} \mathrm{NAg}_{5} \mathrm{I}_{6}$ at $25^{\circ} \mathrm{C}$ is assigned to a relatively small number of mobile $\mathrm{Ag}^{+}$ions. The Hall effect in electrolyte was considered as spurious due to local variations of salt concentration.

\section{Induced Magnetic Field via Chiral Molecules}

\subsection{D Electrochemistry Using Chiral Molecules}

"Three-dimensional (3D) spin-electrochemistry" methodology is based on Hall potential measurements performed in an electrochemical system, where the spin filtering is due to charge transmission, driven electrochemically, through the chiral electrode interface. This leads to spin accumulation, when a suitable long-spin-lifetime material (in this case, a AlGaN/GaN high-electron-mobility transistor) is exploited, resulting in a magnetic field [26-28]. This method provides simultaneously, in operando, a three-way source of information: the applied voltage, the resulting electrochemical current, and spin injection (inducing a magnetic field within the electrode) which is probed via the Hall potential measurement. In particular, the AlGaN/GaN is the working electrode, as in a conventional electrochemical cell, and simultaneously allows for the measurement of the spin polarization of the current, as presented schematically in Figure 5. For this purpose, cyclic 
voltammetry measurements are expedient to force a current flow at the chiral electrode interface (made chiral by adsorption of a polypeptide monolayer). Spin accumulation in the HEMT device originates a relevant magnetic field which is detected by measuring the Hall potential in the current channel, where a constant current is obtained by applying a potential difference between the source and drain (S and D electrodes in Figure 5a) [28]. The Hall potential stems from the spin-polarized electrons injected into (or out from) the GaN device. The accumulated spins in the GaN produce a magnetic field perpendicular to the modified working electrode surface. This method generates the magnetic field via spin filtering obtained by charge transmission through the chiral interface: the CISS effect [5].
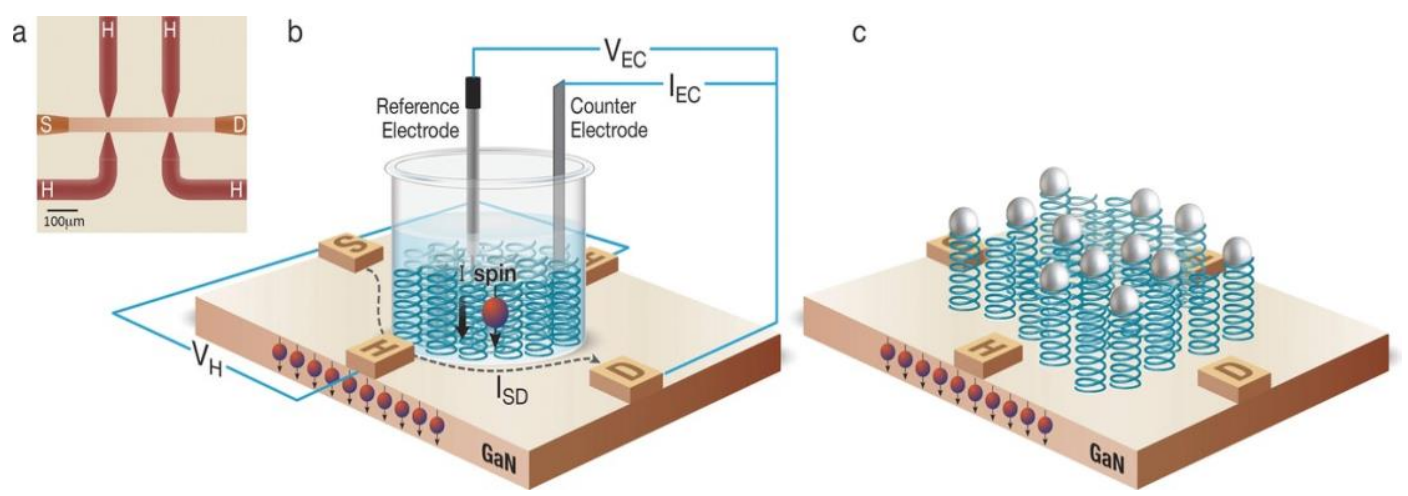

Figure 5. Schematic representation of the experimental setup. (a) Optical image of the GaN-based Hall device. S and D are the source and drain electrodes, respectively, and H marks the tip electrode contacts used for the Hall potential measurement. (b) View of the combined electrochemical/Hall device apparatus. (c) Working electrode covered with Ag nanoparticles, the redox probe, attached to the adsorbed oligopeptide monolayer (Reproduced with permission from [28], WILEY-VCH, 2017).

The measurement of the Hall potential is crucial in detecting the induced magnetic field within the GaN, since it is known that the application of a magnetic field to an electrolytic solution introduces various effects that may influence the global electrochemical behavior of an electrochemical system, even in the absence of any spin-dependent reaction [29], as discussed in previous sections.

This method probes the spin selectivity of electrons transmitted through a monolayer of chiral molecules of various length: the $\alpha$-helical oligopeptides are covalently bound to the surface via the carboxylic group (C-terminal) and to silver clusters (15-20 $\mathrm{nm}$ in diameter) acting as the redox probe. Remarkably, the current in the cyclic voltammograms features quite similar absolute values for all the four adsorbed molecules, while the relevant Hall potential difference increases with the length of the adsorbed molecules. This is due to continuous injection of spin-polarized electrons from the solution to the GaN electrode (compare Figure 6). 

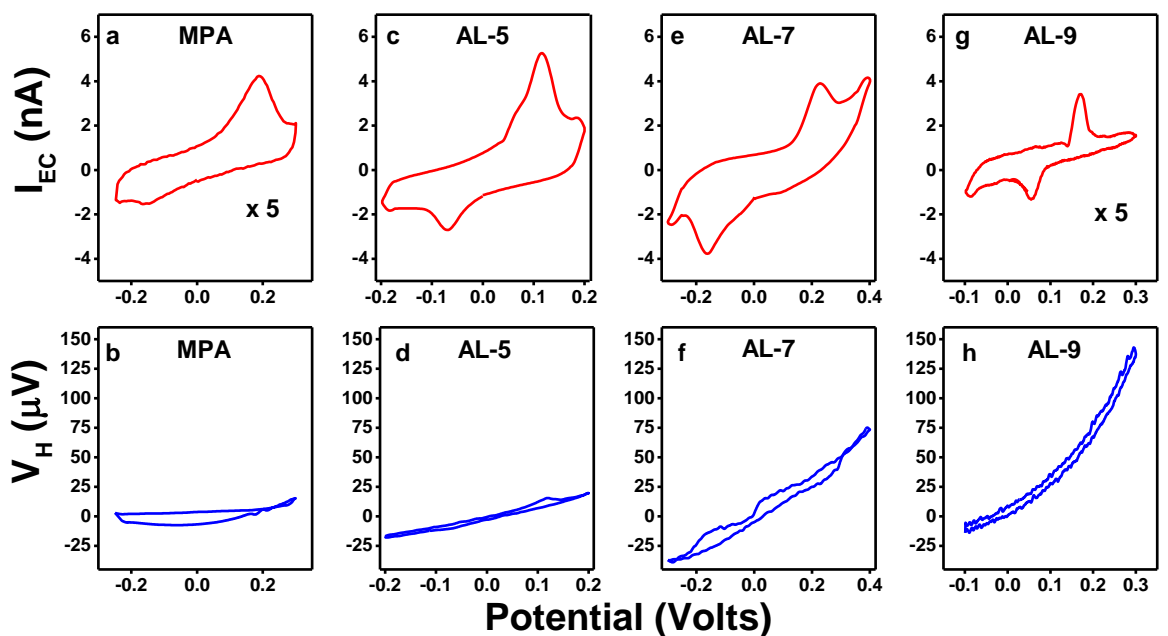

Figure 6. Electrochemical and Hall potential measurements. The reported results are obtained when the GaN device is coated with a self-assembled monolayer of achiral molecules (MPA: 3 mercapto propanoic acid) $(\mathbf{a}, \mathbf{b})$ or with chiral $\alpha$-helical oligopeptides of variable length L-SH-CH $-\mathrm{CH}_{2}-\mathrm{CO}-\{\mathrm{Ala}-\mathrm{Aib}\}_{n}-\mathrm{COOH}$, where $n=5,7$, and 9 for AL-5 (c,d), AL-7 (e,f), and AL-9 $(\mathbf{g}, \mathbf{h})$, respectively (Reproduced with permission from [28], WILEY-VCH, 2017).

The chiral GaN-based working electrode is also exploited to carry out an electrochemically based enantio-recognition study to further investigate the correlation between enantio-selectivity and the spin polarization [6]. To this end, the GaN device was coated with AL-5 and a similar electrochemical experiment, as described above, was performed with a chiral redox couple-either the (S)-(-)-N,N-Dimethyl-1-ferrocenylethylamine or (R)-(+)- $N, N$-Dimethyl-1-ferrocenylethylamine (indicated as S-ferrocene and R-ferrocene, respectively) dissolved in phosphate buffer ( $\mathrm{pH} \sim 7$ ). Since

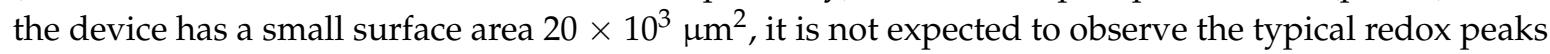
in this type of experiment [30]. Owing to the chiral working electrode interface, an enhanced current is observed in the CV curve with R-ferrocene, as shown in Figure 7c, with higher Hall potential for favorable spin interaction (Figure 7d), while no significant effect is visible in the achiral analogue device (Figure $\mathrm{7b}$ ). The correlation between enantio-selectivity (S- and R-ferrocene redox couple in solution) and the spin polarization (Hall potential) can be inferred by comparing the electrochemical current and Hall potential values as a function of the potential (compare Figure 7c,d graphs).

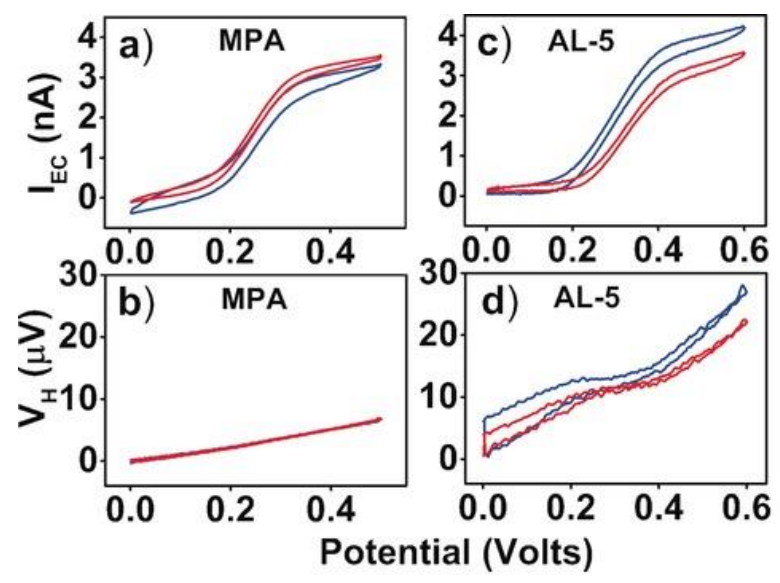

Figure 7. The enantio-selectivity data for devices coated with achiral molecules $(\mathbf{a}, \mathbf{b})$ and with chiral AL-5 (c,d) with chiral R-and S-ferrocene derivatives (blue and red curves, respectively) (Reproduced with permission from [28], WILEY-VCH, 2017). 


\subsection{Magnetic Field Using Electrolytic Gated Chiral Molecules}

Kumar et al. demonstrated experimentally that, in chiral molecules, charge redistribution is accompanied by spin polarization [6]. The spin polarization was measured by using a Hall effect device similar to that described in Section 5.1. An electric field applied along the molecules causes charge redistribution, and for chiral molecules, a Hall voltage is measured. The latter indicates the spin polarization/magnetic field present within the GaN device. Figure 8a shows a schematic representation of the experimental setup. In this spin polarization experiment, a Hall device coated with a self-assembled monolayer of polypeptide was placed in solution. Note that the gate electrode $(G)$ is not in direct contact with the inert electrolyte solution. When an electric potential $V_{G}$ is applied between the $\mathrm{G}$ electrode and the device, a polarization electric field acts on the adsorbed polypeptide. The relevant electrical scheme is shown in Figure $8 \mathrm{~b}$ : $\mathrm{I}_{\mathrm{SD}}$ is the applied $\mathrm{S}-\mathrm{D}$ current, $\mathrm{V}_{\mathrm{G}}$ is the gate voltage, and $\mathrm{V}_{\mathrm{H}}$ is the Hall potential across the conductive channel. As a result, the molecules are polarized because of charge reorganization (partial charges $q+$ and $q-$ ), inducing a charge displacement in the surface region of the device. Because the charge polarization is accompanied by spin polarization (red balls with black arrows, compare Figure 8c), a magnetic field is also created, affecting the electron flow between the $S$ and $D$ electrodes. The Hall potential $V_{H}$, which is formed as a result of the spin magnetization, was measured as a function of $\mathrm{V}_{\mathrm{G}}$.
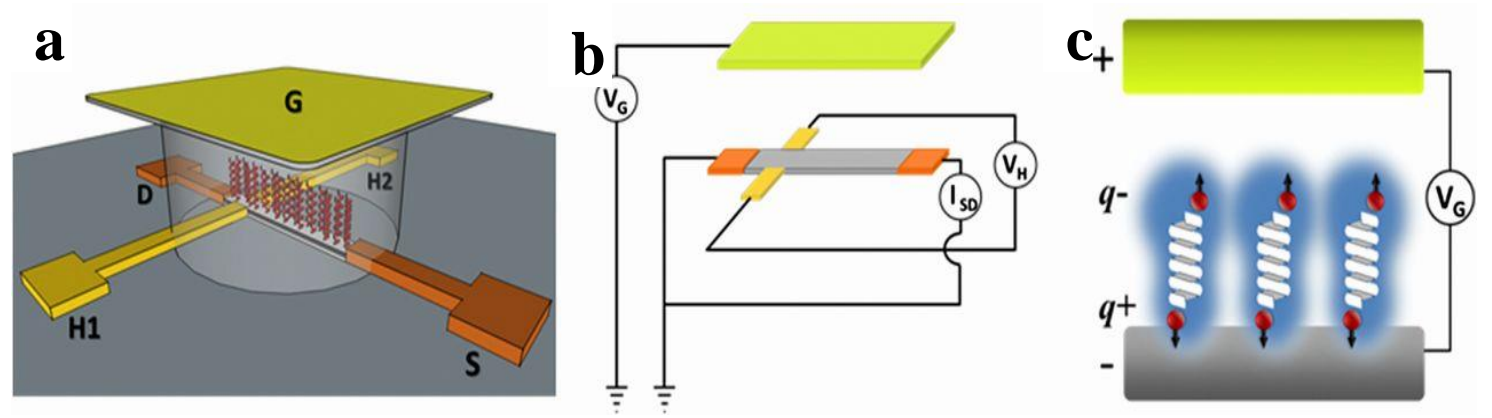

Figure 8. (a) Pictorial view of experimental setup, (b) electrical connections, and (c) concept behind the Hall potential measurements (Reproduced with permission from [6], United States National Academy of Sciences, 2017).

Figure 9a shows the Hall voltages as a function of the gate potential for the chiral oligopeptides: L- and D-SH-CH $-\mathrm{CH}_{2}-\mathrm{CO}-\{\text { Ala-2-aminoisobutyric acid or 2-methylalanine (Aib) }\}_{5}-\mathrm{COOH}$. The Hall voltage observed with the chiral molecules shows a nearly linear dependence as a function of the applied gate voltage; the sign of $\mathrm{V}_{\mathrm{H}}$ depends on the handedness of the chiral adsorbed polypeptide. Figure $9 \mathrm{~b}$ sets out Hall potential $\mathrm{V}_{\mathrm{H}}$ data measured for two chiral L-oligopeptides of different length and an achiral molecule as a control experiment. Because of the high ordered molecular chirality with $\mathrm{L}-\mathrm{SHCH} \mathrm{CH}_{2} \mathrm{CO}-\{\mathrm{Ala}-\mathrm{Aib}\}_{7}-\mathrm{COOH}$, higher spin polarization is created, which is responsible for the higher Hall voltage. For an external voltage of $10 \mathrm{~V}$, the Hall signal corresponds to about a $5 \mathrm{mT}$ field, $\mathrm{SHCH}_{2} \mathrm{CH}_{2} \mathrm{CO}-\{\mathrm{Ala}-\mathrm{Aib}\}_{7}-\mathrm{COOH}$. These results strongly suggest that an effective magnetic field could be generated from the spin-filtering ability of the chiral adsorbed layer. 

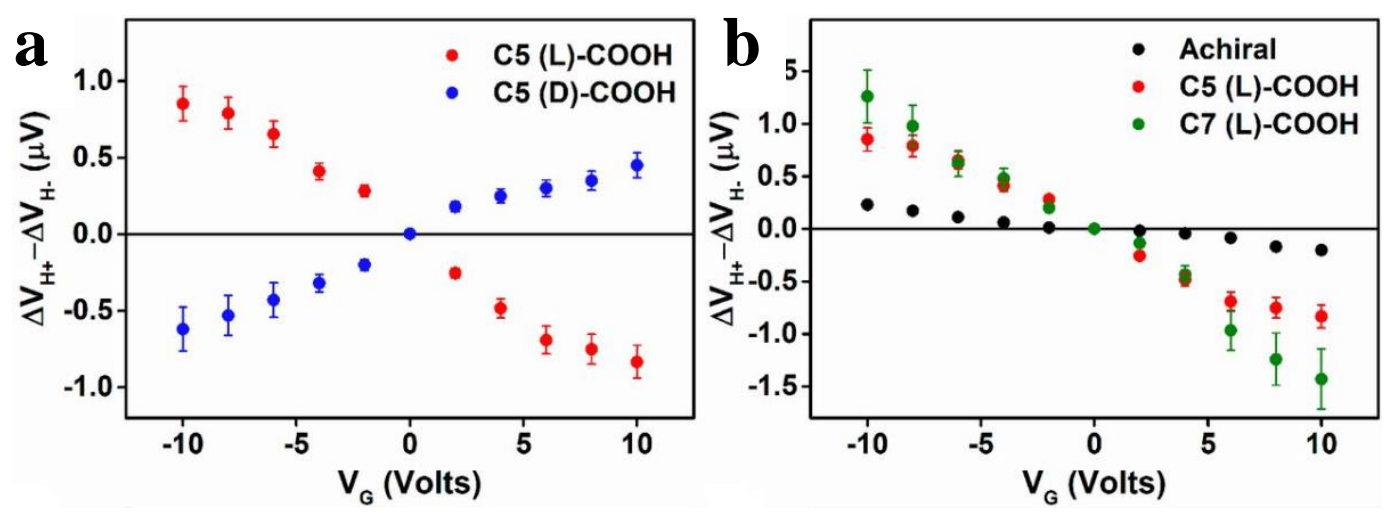

Figure 9. Hall measurements conducted on devices coated with different oligopeptides. (a) Hall potential measured when the adsorbed layer is either L-SH-CH$-\mathrm{CH}_{2}-\mathrm{CO}-\{\mathrm{Ala}-\mathrm{Aib}\}_{5}-\mathrm{COOH}$ (red) or D-SH- $\mathrm{CH}_{2}-\mathrm{CH}_{2}-\mathrm{CO}-\{\text { Ala-Aib }\}_{5}-\mathrm{COOH}$ (blue). (b) The dependence of the Hall voltage on $\mathrm{V}_{\mathrm{G}}$ is shown for monolayer films of achiral 11-mercapto-undecanoic acid (black), chiral $\mathrm{L}-\mathrm{SH}-\mathrm{CH}_{2}-\mathrm{CH}_{2}-\mathrm{CO}-\{\mathrm{Ala}-\mathrm{Aib}\}_{5}-\mathrm{COOH}$ (red), and chiral L-SH-CH$-\mathrm{CH}_{2}-\mathrm{CO}-\{\text { Ala-Aib }\}_{7}-\mathrm{COOH}$ (green) (Reproduced with permission from [6], United States National Academy of Sciences, 2017).

\section{Spin-Controlled Charge Transport in Chiral Molecules}

Study of spin-dependent charge transport across chiral molecular assembly by spin-dependent electrochemistry is the first of its kind, specifically designed for measuring spin polarization. Prof Naaman's group established this state-of-the art technique in which chiral molecules are deposited directly onto a ferromagnetic working electrode. The latter can be magnetized with its magnetic moment either pointing "UP" or "DOWN" by placing a permanent magnet underneath the ferromagnetic electrode. The direction of the magnet can be flipped from "UP" to "DOWN" or vice-versa to obtain UP or DOWN spin-polarized electron injection; thus a Faradic current can be measured via cyclic voltammetry measurements under two different spin polarization regimes. Eventually the relevant electrochemical spin-polarized current can be measured. Again, as in ref. [28], charge transmission through the chiral electrode/solution interface is driven by an electrochemical charge transfer process: a suitable redox probe is present in bulk solution or immobilized on the electrode surface. To study the spin-dependent electrochemical charge transfer process, a nickel ferromagnetic working electrode is used as a spin injector [31]. The high chemical affinity of nickel towards oxygen calls for great care in the adsorption of organic compounds (thiols in particular)—such a process is hindered by the spontaneous nickel oxide formation [32]. To overcome such a problem, an original procedure has been established to allow for simultaneous electrochemical reduction of nickel and adsorption of thiols (chiral) molecules [32]. In a typical spin-dependent electrochemistry study, a chiral polymer resulting in about a $\sim 3 \mathrm{~nm}$ organic film thickness, poly\{[methyl $N$-(tert-butoxycarbonyl)-S-3-thienyl-L-cysteinate]-cothiophene\} (compare Figure 10a), was adsorbed on the nickel surface. Then, the Faradic current (the ferrocene redox probe was present in solution) was determined by cyclic voltammetry measurements, under magnet "UP" and "DOWN" configurations (Figure 10b). The spin-dependent charge transfer results are compelling: when the chiral-polymer-coated Ni electrode was magnetized in the "UP" direction, the Faradic current changed its direction during oxidation to a reduction process (red curve, Figure 10c). On the other hand, when the Ni working electrode was magnetized in the "DOWN" direction, the cathodic current did not change its sign with reference to the anodic process. Thus, the "DOWN" spin-controlled electron transfer process reveals a relatively higher energy barrier for electron conduction to occur from the nickel working electrode to the oxidized ferrocene molecules present in an electrolyte (black curve, Figure 10c). Thus a thin layer of chiral conductive polymer acts as an excellent spin filter which allows the transmission of only one type of spin [33]. 

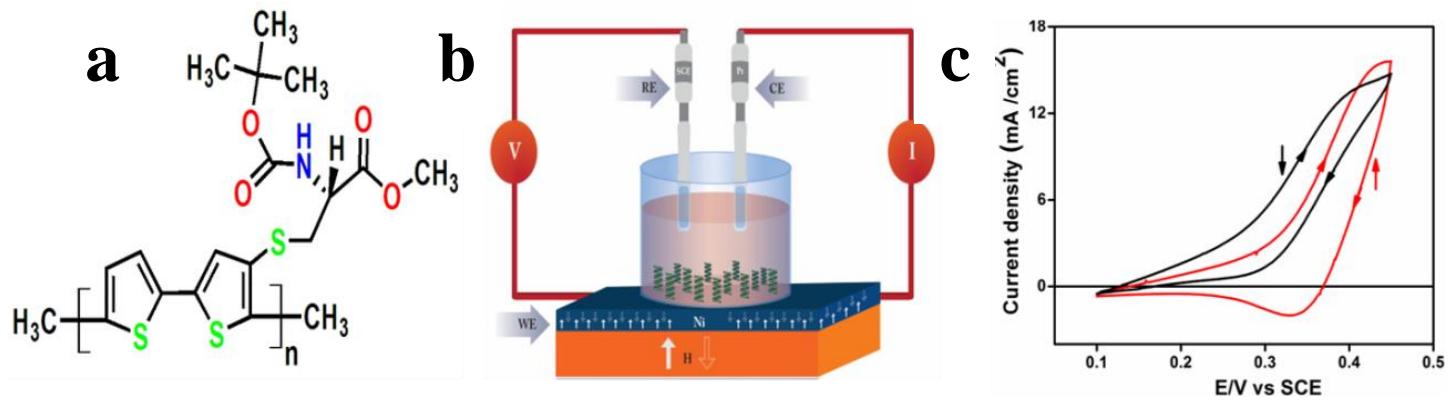

Figure 10. (a) Poly\{[methyl N-(tert-butoxycarbonyl)-S-3-thienyl-L-cysteinate]-cothiophene $\}$ chemical structure and (b) schematic representation of the spin-dependent electrochemistry setup. The ferromagnetic Ni was coated with chiral polymers and used as the working electrode which can be magnetized by an external magnetic field $(\mathrm{H})$. (c) Spin-dependent cyclic voltammograms recorded on the chiral-polymer-coated Ni working electrode in the presence of achiral ferrocene redox probe measured under magnetic field pointing "UP" (solid red curve) and "DOWN" (solid black curve) by applying a static magnetic field of $0.35 \mathrm{~T}$ (Reproduced with permission from [33], WILEY-VCH, 2015).

An exciting variation is to perform spin-dependent experiments involving biologically important macromolecules, such as oligopeptides or redox proteins. It is virtually impossible to immobilize these biological systems onto a ferromagnetic electrode via simple in situ electrochemical reduction methods. This is because of the high negative potential needed to reduce the nickel oxide, which may damage or denature the biologically important macromolecules. In a recent study, an alternative way to prevent the oxide formation of the Ni electrode while allowing for the production of a surface suitable for Ni surface functionalization has been proposed [34]. This result is obtained by growing a thin Au overlayer (in the 5 to $15 \mathrm{~nm}$ range) on top of the Ni substrate without breaking the vacuum during the deposition process. Ni covered with an ultrathin Au overlayer is much more stable and was used to prepare self-assembled monolayers (SAMs) of L- and D-cysteine. The functional SAMs having free $-\mathrm{NH}_{2}$ groups were employed to make a covalent bond with an organic redox probe, toluidine blue $\mathrm{O}$ (TBO) (compare Figure 11a), in order to measure Faradic current under an applied magnetic field of $0.35 \mathrm{~T}$. Interestingly, two enantiomeric forms of TBO-Cys (L and D) showed opposite spin polarization (Figure 11b,c). D-Cys-TBO assembly shows spin-dependent Faradaic current, and the spin polarization was measured at $-6 \%$ for the oxidation peak potential at $+0.185 \mathrm{~V}$ and $+4.7 \%$ for the reduction process at $-0.056 \mathrm{~V}$ vs. standard calomel electrode (SCE) (Figure 11b). The L-Cys-TBO chiral assembly shows reverse spin polarization measured at $+9 \%$ for the oxidation process at $0.160 \mathrm{~V}$, while the spin polarization (SP) was at $-7.5 \%$ found at the reduction process at $-0.024 \mathrm{~V}$ (Figure 11c). Spin-dependent cyclic voltammograms of L-Cys-TBO were recorded for increasing Au overlayer thickness and showed a neat decrease in spin polarization upon increasing thickness of the gold overlayer on top of the Ni electrode (the spin coherence of the electrons transmitted through a thick layer is destroyed by the strong spin-orbit coupling property of the gold). 

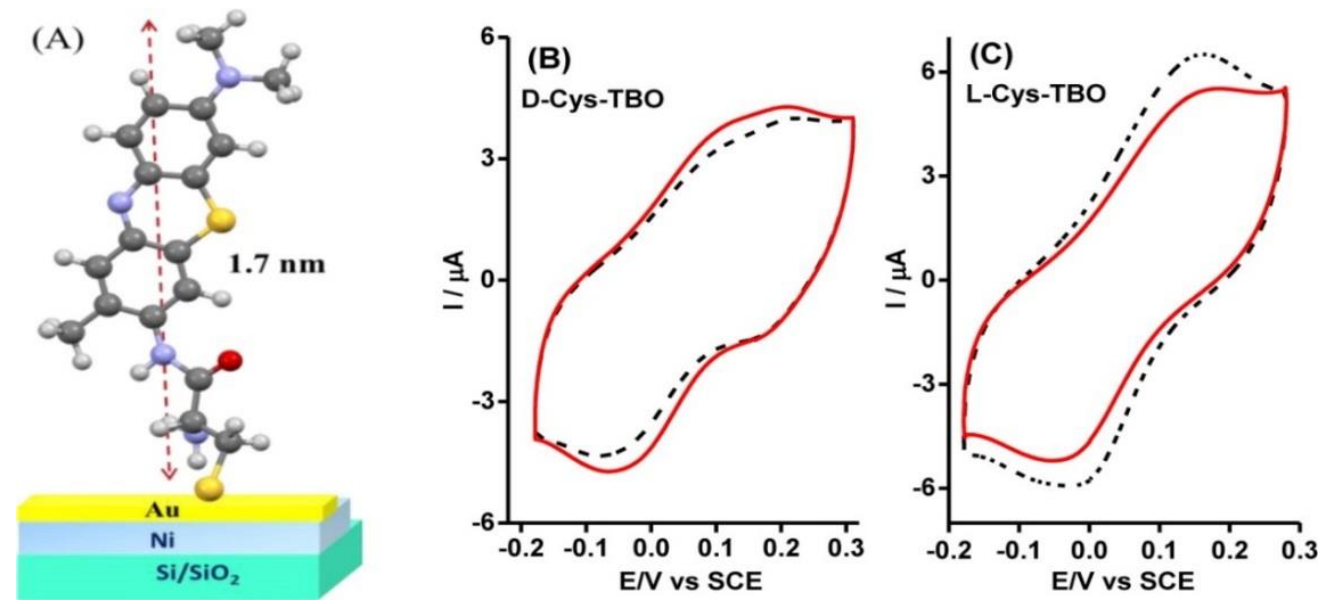

Figure 11. (a) Schematic description of covalently bonded toluidine blue $\mathrm{O}$ (TBO) to the self-assembled monolayers (SAMs) of L or D cysteine prepared on $10 \mathrm{~nm}$ Au-coated Ni working electrode, (b) spin-dependent cyclic voltammograms of D-cys-TBO, and (c) L-cys-TBO self-assembly grafted on Au-overlayer-coated Ni electrode. The voltammograms were recorded in $0.1 \mathrm{M}$ PBS of pH 7.4 containing $10 \mathrm{mM} \mathrm{KCl}$ as supporting electrolyte at $50 \mathrm{mV} \mathrm{s}^{-1}$ (Reproduced with permission from [34], American Chemical Society, 2015).

\section{Conclusions}

This review focuses on magnetic field effects in different areas of magneto-electrochemistry. Electrodeposition and electropolymerization processes, electrolyte bulk charge transport, and the quite recent spin-dependent electrochemistry are the topics here considered. In particular, spin-dependent electrochemistry, which is based on the interplay between chirality/spin/magnetic field physical quantities [35], seems promising for appealing applications in sensor- and adsorption-related (chromatography in primes) technological areas. Remarkably, recent results show that chirality is able, via the CISS effect, to drive spin-selective electron transfer allowing the control of local magnetic fields, making it suitable for molecular-based spintronic devices.

It is worth emphasizing the fact that, at present, phenomena and experiments reported in Sections 3 and 4 are apparently unrelated to the spin-dependent electrochemistry approach. However, these two disjoined fields of magnetochemistry can find a possible overlap, like, for instance, the use of chiral electrodeposited metals or alloys in spin-dependent electrochemistry. This will possibly allow for the emergence of new unexplored synergic effects in both chiral recognition and spin injection.

Acknowledgments: P.C.M. thanks the European Union for Marie-Curie Fellowship (H2020-MSCA-2015-706238). C.F. acknowledges financial support for this research by University of Modena and Reggio Emilia (Department of Engineering 'Enzo Ferrari'), through "Spin Dependent Electrochemistry", FAR2016.

Conflicts of Interest: The authors declare no conflict of interest.

\section{References}

1. Coey, J.M.D.; Hinds, G. Magnetoelectrolysis-the effect of magnetic fields in electrochemistry. In Proceedings of the 5th International Pamir Conference, Ramatuelle, France, 16-20 September 2002; pp. 1-7.

2. Fahidy, T.Z. Magnetoelectrolysis. J. Appl. Electrochem. 1983, 13, 553-563. [CrossRef]

3. Bund, A.; Koehler, S.; Kuehnlein, H.H.; Plieth, W. Magnetic field effects in electrochemical reactions. Electrochim Acta 2003, 49, 147-152. [CrossRef]

4. Fahidy, T.Z. Characteristics of surfaces produced via magnetoelectrolytic deposition. Prog. Surf. Sci. 2001, 68, 155-188. [CrossRef]

5. Naaman, R.; Waldeck, D.H. Chiral-Induced Spin Selectivity Effect. J. Phys. Chem. Lett. 2012, 3, $2178-2187$. [CrossRef] [PubMed] 
6. Kumar, A.; Capua, E.; Kesharwani, M.K.; Martin, J.M.L.; Sitbon, E.; Waldeck, D.H.; Naaman, R. Chirality-induced spin polarization places symmetry constraints on biomolecular interactions. Proc. Natl. Acad. Sci. USA 2017, 114, 2474-2478. [CrossRef] [PubMed]

7. Shi, X.; Wang, Y.; Peng, C.; Zhang, Z.; Chen, J.; Zhou, X.; Jiang, H. Enantiorecognition of Tyrosine Based on a Novel Magnetic Electrochemical Chiral Sensor. Electrochim Acta 2017, 241, 386-394. [CrossRef]

8. Mishra, D.; Markus, T.Z.; Naaman, R.; Kettner, M.; Gohler, B.; Zacharias, H.; Friedman, N.; Sheves, M.; Fontanesi, C. Spin-dependent electron transmission through bacteriorhodopsin embedded in purple membrane. Proc. Natl. Acad. Sci. USA 2013, 110, 14872-14876. [CrossRef] [PubMed]

9. Yu, P.; Cardona, M. Fundamentals of Semiconductors: Physics and Materials Properties, 4th ed.; Graduate Texts in Physics; Springer: Berlin/Heidelberg, Germany, 2010; ISBN 978-3-642-000709-5.

10. Alfven, H. Existence of Electromagnetic-Hydrodynamic Waves. Nature 1942, 150, 405-406. [CrossRef]

11. Rhen, F.M.F.; Coey, J.M.D. Magnetic Field Effect on Autocatalysis: Ag and Cu in Concentrated Nitric Acid. J. Phys. Chem. B 2006, 110, 6274-6278. [CrossRef] [PubMed]

12. Mogi, I.; Morimoto, R.; Aogaki, R.; Watanabe, K. Surface chirality induced by rotational electrodeposition in magnetic fields. Sci. Rep. 2013, 3, 2574. [CrossRef] [PubMed]

13. Mhíocháin, T.R.N.; Coey, J.M.D. Chirality of electrodeposits grown in a magnetic field. Phys. Rev. E $2004,69$. [CrossRef] [PubMed]

14. Ispas, A.; Matsushima, H.; Plieth, W.; Bund, A. Influence of a magnetic field on the electrodeposition of nickel-iron alloys. Electrochim Acta 2007, 52, 2785-2795. [CrossRef]

15. Mogi, I.; Watanabe, K. Electrocatalytic Chirality on Magneto-Electropolymerized Polyaniline Electrodes. Available online: http:/ / www.ingentaconnect.com/content/ssam/14328488/2007/00000011/00000006/ art00010;jsessionid=bchasa5ilpue2.x-ic-live-01 (accessed on 1 August 2018).

16. Horvath, J.D.; Koritnik, A.; Kamakoti, P.; Sholl, D.S.; Gellman, A.J. Enantioselective Separation on a Naturally Chiral Surface. J. Am. Chem. Soc. 2004, 126, 14988-14994. [CrossRef] [PubMed]

17. Lorenzo, M.O.; Baddeley, C.J.; Muryn, C.; Raval, R. Extended surface chirality from supramolecular assemblies of adsorbed chiral molecules. Nature 2000, 404, 376-379. [CrossRef] [PubMed]

18. Horvath, J.D.; Gellman, A.J. Enantiospecific Desorption of Chiral Compounds from Chiral Cu(643) and Achiral Cu(111) Surfaces. J. Am. Chem. Soc. 2002, 124, 2384-2392. [CrossRef] [PubMed]

19. Switzer, J.A.; Kothari, H.M.; Poizot, P.; Nakanishi, S.; Bohannan, E.W. Enantiospecific electrodeposition of a chiral catalyst. Nature 2003, 425, 490-493. [CrossRef] [PubMed]

20. Xie, Y.; Huber, C.O. Electrocatalysis and amperometric detection using an electrode made of copper oxide and carbon paste. Anal. Chem. 1991, 63, 1714-1719. [CrossRef]

21. Hinds, G.; Spada, F.E.; Coey, J.M.D.; Ní Mhíocháin, T.R.; Lyons, M.E.G. Magnetic Field Effects on Copper Electrolysis. J. Phys. Chem. B 2001, 105, 9487-9502. [CrossRef]

22. Wattanakit, C.; Côme, Y.B.S.; Lapeyre, V.; Bopp, P.A.; Heim, M.; Yadnum, S.; Nokbin, S.; Warakulwit, C.; Limtrakul, J.; Kuhn, A. Enantioselective recognition at mesoporous chiral metal surfaces. Nat. Commun. 2014, 5, 3325. [CrossRef] [PubMed]

23. Frank, R.L.; Hoffman, J.G. Hall voltage in electrolyte solutions. Phys. Chem. Liq. 1972, 3, 191-204. [CrossRef]

24. Hubbard, J.B.; Wolynes, P.G. An electrohydrodynamic contribution to the Hall effect in electrolyte solutions. J. Chem. Phys. 1981, 75, 3051-3054. [CrossRef]

25. Newman, D.S.; Frank, C.; Matlack, R.W.; Twining, S.; Krishnan, V. The ionic hall effect in the solid electrolyte $\mathrm{C}_{5} \mathrm{H}_{6} \mathrm{NAg}_{5} \mathrm{I}_{6}$. Electrochim Acta 1977, 22, 811-814. [CrossRef]

26. Hall, E.H. On a New Action of the Magnet on Electric Currents. Am. J. Math. 1879, 2, 287-292. [CrossRef]

27. Eckshtain-Levi, M.; Capua, E.; Refaely-Abramson, S.; Sarkar, S.; Gavrilov, Y.; Mathew, S.P.; Paltiel, Y.; Levy, Y.; Kronik, L.; Naaman, R. Cold denaturation induces inversion of dipole and spin transfer in chiral peptide monolayers. Nat. Commun. 2016, 7. [CrossRef] [PubMed]

28. Kumar, A.; Capua, E.; Vankayala, K.; Fontanesi, C.; Naaman, R. Magnetless Device for Conducting Three-Dimensional Spin-Specific Electrochemistry. Angew. Chem. 2017, 129, 14779-14782. [CrossRef]

29. Monzon, L.M.A.; Coey, J.M.D. Magnetic fields in electrochemistry: The Lorentz force. A mini-review. Electrochem. Commun. 2014, 42, 38-41. [CrossRef]

30. Walsh, D.A.; Lovelock, K.R.J.; Licence, P. Ultramicroelectrode voltammetry and scanning electrochemical microscopy in room-temperature ionic liquid electrolytes. Chem. Soc. Rev. 2010, 39, 4185-4194. [CrossRef] [PubMed] 
31. Mondal, P.C.; Fontanesi, C.; Waldeck, D.H.; Naaman, R. Spin-Dependent Transport through Chiral Molecules Studied by Spin-Dependent Electrochemistry. Acc. Chem. Res. 2016, 49, 2560-2568. [CrossRef] [PubMed]

32. Fontanesi, C.; Tassinari, F.; Parenti, F.; Cohen, H.; Mondal, P.C.; Kiran, V.; Giglia, A.; Pasquali, L.; Naaman, R. New One-Step Thiol Functionalization Procedure for Ni by Self-Assembled Monolayers. Langmuir 2015, 31, 3546-3552. [CrossRef] [PubMed]

33. Mondal, P.C.; Kantor-Uriel, N.; Mathew, S.P.; Tassinari, F.; Fontanesi, C.; Naaman, R. Chiral Conductive Polymers as Spin Filters. Adv. Mater. 2015, 27, 1924-1927. [CrossRef] [PubMed]

34. Mondal, P.C.; Fontanesi, C.; Waldeck, D.H.; Naaman, R. Field and Chirality Effects on Electrochemical Charge Transfer Rates: Spin Dependent Electrochemistry. ACS Nano 2015, 9, 3377-3384. [CrossRef] [PubMed]

35. Fontanesi, C. Spin-dependent electrochemistry: A novel paradigm. Curr. Opin. Electrochem. 2018, 7, 36-41. [CrossRef]

(C) 2018 by the authors. Licensee MDPI, Basel, Switzerland. This article is an open access article distributed under the terms and conditions of the Creative Commons Attribution (CC BY) license (http://creativecommons.org/licenses/by/4.0/). 